\title{
Incidencia de dos modelos de competición de fútbol sobre los valores socio-educativos en prebenjamines \\ The impact of two models of football competition on social and educational values in Under-eights \\ Rubén Veroz Domínguez*, José María Yagüe Cabezón**, Belén Tabernero Sánchez*** \\ *C.P. Valdellera de Posada de Llanes (España), **Universidad de León (España), ***Universidad de Salamanca (España)
}

\begin{abstract}
Resumen. La enseñanza del deporte base debería perseguir la transmisión de valores educativos. El artículo tiene como objetivo principal analizar el proceso de asimilación de algunos de estos valores socio-educativos (autonomía responsable, respeto, prosocialidad y nivel de satisfacción-diversión) en un contexto de competición mediante la aplicación del cuestionario «Deporteduca». En el estudio han intervenido 467 participantes divididos en tres grupos: niños de entre cuatro y siete años, sus padres y formadores. Estos futbolistas participaban en dos modelos de competición diferentes: ligas federativas de fútbol 7 y competición educativa «Futgolines». En este trabajo se comparan las medias obtenidas en cada grupo de participantes de los dos modelos de competición, y se valora si el resultado es significativo empleando el índice Chi-cuadrado $\left(\chi^{2}\right)$. En ambos modelos de competición se perciben mejoras en la adquisición de los valores cuantificados, pero existen diferencias estadísticamente significativas en la dimensión «Autonomía responsable y respeto» a favor del modelo «Futgolines». Se obtienen datos similares en la categoría de «Satisfacción-diversión» y ligeramente superiores entre los participantes en el modelo federativo en la dimensión «Prosocialidad».
\end{abstract}

Palabras claves. Valores, competición, fútbol, iniciación, agentes participantes.

Abstract. The methodology used in junior sports must focus on communicating educational values. This paper aims to analyze the assimilation of these educational values (responsible autonomy, respect, prosocial behavior and degree or satisfaction/ enjoyment) in a competitive context, by using the «Deporteduca» questionnaire. The questionnaire was administered to 467 participants divided into three groups: children aged four-seven, their parents, and their trainers. These football (soccer) players took part in two different competition models: seven-a-side federation football (soccer) leagues and the «Futgolines» alternative model of educational competition. This research compares the individual and average results obtained by participants in the two different formats and assesses whether these results are related using Chi-square $\left(\chi^{2}\right)$. Both competition models showed an improvement in the acquisition of the quantified values, but the results show significant higher average values among «Futgolines» participants in the categories of «responsible autonomy and respect». The results in the «satisfaction/enjoyment» category are similar and those in the "prosocial behavior» category are slightly higher in the federative model.

Key words. Values, competition, football, initiation, players concerned.

\section{Introducción}

La competición forma parte del deporte. Sin embargo, el carácter formativo de la competición ha sido cuestionado por numerosos autores (Mandado \& Díaz, 2004, Gutiérrez \& Pilsa, 2006; Hidalgo, 2008). Parece necesario observar dos condiciones para que la competición resulte beneficiosa, debe estar orientada a la aceptación de las normas y a la cooperación (Durán, 2013). Pero a su vez, la competición, representa un contexto real en el que aparecen sensaciones como alegría, satisfacción, cooperación, respeto hacía sí mismo y hacia los demás, empatía, altruismo... y al mismo tiempo otras como intensidad, emociones y actitudes como el enfado, el resentimiento, la rivalidad, la ofuscación o la agresividad (Ruiz, 2004). La propia experimentación y adecuada gestión de estas sensaciones que se viven en el momento de la competición, podrá provocar un progreso a nivel deportivo, y quizás un desarrollo a nivel personal.

El tratamiento de la competición deportiva en elámbito de la iniciación deportiva queda regulado por el Consejo Superior de Deportes en su Plan Integral de la Actividad Física y el Deporte (2010) donde se indica que hay que «adaptar las competiciones», siendo necesario concebir un «modelo propio no mimético de la competición federada» que «garanticen el carácter educativo en las competiciones deportivas en edad escolan».

Existe abundante bibliografía que relaciona la competición con la adquisición de valores (López, 2005; Piñar, Cárdenas, Miranda \& Torre, 2008; Gutiérrez, Carratalá, Guzmán \& Pablos, 2010). En este sentido, conviene aclarar el concepto de valor y otros términos que pueden también intervenir cuando se analiza el fenómeno de la competición deportiva. Los valores son creencias personales sobre la adecuación de una serie de conductas que determinan el comportamiento. Los valores analizados en este trabajo han sido seleccionados tomando como base los paradigmas teóricos propuestos por Erikson (1971) y Kohlberg (1982) Por ello, se ha partido de los planteamientos que se demandan

Fecha recepción: 22-09-14- Fecha envío revisores: 30-09-14- Fecha de aceptación: 10-01-15 Rubén Veroz Domínguez rubenveroz@gmail.com para la competición deportiva en la etapa de formación, sirviéndonos de ellos para definir los valores socio-educativos que han sido evaluados:

Autonomía responsable: Actuación de los niños adecuada a la normativa de juego y orientada hacia un compromiso colectivo, al que se llega sin perder grados de libertad personal, y que empieza a construir consciencia de las consecuencias de sus acciones (Barberá, 2001; Marina, 2004; Sánchez-Oliva, Leo, Sánchez-Miguel, Amado \& García-Calvo, 2012)

Respeto (subordinado al anterior en forma): Disposición hacia el cuidado y atención de las personas y las normas concebidas como necesarias para un desarrollo colectivo (Chappuis \& Thomas, 1989; López, 2005).

Prosocialidad: Preocupación activa por el bienestar de otras personas -afecto, capacidad para perdonar, lealtad, amistad- (Schwartz, Verkasalo, Antonovsky \& Sagiv, 1997).

Satisfacción- diversión: Disfrute por la motivación intrínseca suscitada hacia la práctica deportiva (Sánchez, Yagüe, \& Molinero, 2012; Sánchez-Oliva et al., 2012), que derivará en ganas de seguir participando (adherencia), producto de la satisfacción por lo experimentado (Valero, Conde, Delgado, \& Conde, 2004).

Estos valores descritos han sido medidos mediante un cuestionario «Deporteduca» que fue previamente diseñado y validado desde la competición de fútbol en sujetos de temprana edad. Dicho cuestionario, permite la triangulación de la información ya que está adaptado a niños, padres y formadores. Además se ha creado un modelo de competición alternativo a los modelos federativos: «Futgolines» que se basa fundamentalmente en la adaptación del reglamento priorizando evidencias de desarrollo educativo-social.

Al no haberse llevado a cabo estudios científicos para evaluar la adquisición de valores en niños participantes en estos modelos competitivos, en el presente estudio nos planteamos el siguiente objetivo: Analizar el modelo de competición «Futgolines» comprobando la capacidad intrínseca del formato para que los participantes desarrollen los valores de autonomía responsable, respeto, prosocialidad y satisfacción-diversión. A su vez, comparar los resultados con los obtenidos en un modelo de competición estandarizado desde el marco federativo. 


\section{Metodología}

\section{Procedimiento de la investigación}

La presente investigación se ha desarrollado utilizando el momento de la competición deportiva en fútbol. En la iniciación deportiva es habitual el uso del modelo federativo propuesto por la Federación de Fútbol para competir. En esta investigación, sin embargo, se ha creado un modelo de competición alternativo denominado «futgolines», que se basa fundamentalmente en la adaptación del reglamento priorizando evidencias de desarrollo educativo-social. ${ }^{1}$

El inicio de la investigación comenzó con la selección de la muestra. Para ello, se contactó con los responsables de los 37 equipos participantes en competición deportiva siguiendo el modelo «Futgolines». Fueron informados del propósito de la investigación y el procedimiento a seguir. Se realizó una aceptación por escrito de cada Escuela o Club. Así mismo, se informó a padres y se solicitó una autorización por escrito del interés de participar en la investigación y un permiso de participación de sus hijos.

El cuestionario se administró en primer lugar a padres (FUTpadres) que aceptaban la participación de sus hijos (FUTniños) en la investigación y posteriormente a los futbolistas ${ }^{2}$. El tercer grupo de sujetos en rellenar el cuestionario fueron los formadores (FUTformadores) de los equipos que estaban participando en el estudio.

El muestreo en la población (futbolistas) de «Futgolines», es probabilístico aleatorio simple (por estar presente únicamente en localidades concretas donde se ha implantado dicho modelo), de manera que se puede afirmar que nuestra estimación es no sesgada y tenemos un 95\% de confianza en que nuestra estimación se encontrará dentro de dos puntos porcentuales alrededor de la proporción verdadera(Scheaffer, Mendenhal \& Lyman, 2006). Condicionada por esta población, se ha limitado la población de estudio dentro del modelo federativo, procurando así la coincidencia contextual de los participantes en el cuestionario desde los dos formatos, siendo éste un muestreo no probabilístico de carácter opimático (Sierra-Bravo, 1996; Scheaffer, Mendenhal \& Lyman, 2006) o deliberado (Buendía, Colás \& Hernández, 1998).

Por ello, la muestra «Futgolines», fue determinante para el tamaño de la muestra en el grupo competición según el modelo federativo. Siguiendo el mismo protocolo explicado anteriormente se procedió a la cumplimentación de los cuestionarios por parte de niños (FEDniños), padres (FEDpadres) y formadores (FEDformadores) del modelo de competición federativo. Del mismo modo, se procedió a solicitar el consentimiento y aceptaciones oportunas para participar en la investigación.

Tras obtener los cuestionarios rellenados por todos los participantes, se procedió al análisis estadístico de los mismos realizándolo como se describe a continuación. Además se realizaron otros procesos de triangulación de la información ${ }^{3}$ (grupos de discusión y filmación en video de competiciones)

\section{Participantes}

La muestra participante en el estudio ha estado formada por 208 niños, con edades entre cuatro y siete años ( $\mathrm{M}=6.3$; $\mathrm{DT}=.89$ ), que se distribuyeron en dos grupos según el modelo de competición en el que participaron: «Futgolines» -FUT- (M=5.9), con 120 participantes o federativo -FED- (M=6.8), con 88 participantes. Además en el estudio participaron 221 padres (139 FUT y 82 FED) y 38 formadores (17 FUT y 21 FED) vinculados a ambos grupos de futbolistas (Tabla 1).

\section{Instrumentos}

El cuestionario «Deporteduca» consiste en un instrumento de 24 preguntas con triple vía de recogida de información.

La validez de contenido se analizó a través de la consulta tanto cualitativa como cuantitativa (Lawshe, 1975), a doce expertos cuyas aportaciones contribuyeron a la construcción del cuestionario definitivo. La validez de constructo se analizó tomando el índice de correlación Rho de Spearman, cuyos valores determinaron la división de los diferentes ítems en las correspondientes dimensiones. Asu vez se realizó el análisis factorial exploratorio de componentes principales con rotación Varimax, la medida de adecuación del muestreo de Kaiser-Meyer-Olkin (KMO) y la prueba de esfericidad de Bartlet (López, Pérez \& Ramos, 2011). A través del Coeficiente Alfa de Cronbach se comprobó la consistencia interna, que sumado a la prueba test-retest y sirviéndonos del índice de concordancia Kappa de Cohen, se manifestaron niveles adecuados de fiabilidad (Celina \& Campo, 2005).

\section{Análisis estadístico}

En primer lugar, se agrupan los ítems de cada grupo destinatario (niños, padres y formadores) según las dimensiones analizadas (autonomía responsable y respeto, prosocialidad y satisfacción-diversión). De cada conjunto de ítems resultante, realizamos los estadísticos descriptivos para valorar si las respuestas seleccionadas están orientadas o no hacia el desarrollo de los mencionados valores y se realiza un análisis comparativo entre los dos grupos sometidos a estudio.

Para la obtención de los resultados pretendidos en el presente trabajo, han sido realizados estadísticos descriptivos de cada uno de los ítems con la finalidad de describir la distribución de los datos (SPSS 15.0). Fue comparado el porcentaje de respuesta en cada uno de los ítems, así como los resultados medios. Sirviéndonos del índice Chicuadrado $\left(\chi^{2}\right)$, se han comparado los resultados obtenidos en las respuestas de niños, padres y formadores, que están inmersos en el modelo de competición Futgolines, con aquellos que juegan bajo el modelo imperante de la Federación, utilizando el criterio de $p<.05$ para determinar la significación estadística.

\section{Resultados}

Los resultados de la investigación proceden de las opiniones vertidas por los participantes a través de la aplicación del cuestionario «Deporteduca». Para una mayor claridad, se mostrarán dichos resultados estructurados según la pertenencia de los ítems a cada una de las dimensiones analizadas.

Como puede verse en la tabla 2, las dos primeras dimensiones "Autonomía responsable y respeto» y «Prosocialidad», son operativas con nueve ítems, quedando seis preguntas para aportar información relativa a la tercera dimensión analizada «Satisfacción-diversión». Las respuestas, inicialmente de carácter nominal, fueron ubicadas en una escala ordinal lógica. Se otorgaron valores de 1 a 4, alternándose el sentido de las mismas para que en unas ocasiones la opción más positiva estuviera sujeta a la respuesta número 1 y en otras, a la respuesta 4.

Las dimensiones de autonomía responsable y respeto son valoradas con tres preguntas de las cuáles, las dos primeras están más orientadas hacia la primera de las variables, mientras que la tercera ofrece información más vinculada al respeto, encuadrándose en una misma categoría por la interdependencia de ambos contenidos. El matiz de respeto al reglamento se encuentra directamente vinculado con la responsabilidad, por lo que permite recoger información del desarrollo de ambos valores. En el caso de la prosocialidad, se obtienen datos en tres peguntas en cada cuestionario, mientras que la dimensión de satisfacción / diversión es valorada únicamente con dos preguntas (tabla 2). El orden de las preguntas no coincide exactamente para alternar las diferentes dimensiones, no considerándose tan necesario en el caso de los niños.

Resultados en la dimensión «Autonomía responsable y respeto» Esta categoría está formada por los ítems 1, 2 y 4 en las preguntas destinadas para niños, y los ítems 1, 2 y 6 para las preguntas realizadas a padres y formadores. En todos estos ítems el valor que predice mayor desarrollo de los valores analizados es 1 . Del modelo de competición «Futgolines» fueron completados 276 cuestionarios, obteniéndose valores medios de respuesta de 1.55, mientras que entre los 191 participantes consultados de «Federación», el valor medio ascendió a 1.72 (Tabla 3). Comparamos estos resultados con los obtenidos en las respuestas de los participantes en el formato federativo:

El primer ítem es relativo a la voluntariedad de participación de los 
Tabla 1

\begin{tabular}{|c|c|c|c|}
\hline & Futgolines & Federación & Total \\
\hline Niños & 120 & 88 & 208 \\
\hline Padres & 139 & 82 & 221 \\
\hline Formadores & 17 & 21 & 38 \\
\hline Total & 276 & 191 & 467 \\
\hline \multicolumn{4}{|c|}{ re dimensiones y preauntas on los tres cuest } \\
\hline \multirow{2}{*}{ Dimensiones } & \multicolumn{3}{|c|}{ Items } \\
\hline & Niños & Padres & Form adores \\
\hline Aut. responsable y respet o & $1,2,4$ & $1,2,6$ & $1,2,6$ \\
\hline Prosociliadad & $3,5,6$ & $3,5,7$ & $3,5,7$ \\
\hline Satisfacción - diversión & 7,8 & 4,8 & 4,8 \\
\hline
\end{tabular}

Tabla3

Es tadísticos descriptivos ítems de la dimen sión "Autonomía responsable y respeto"

\begin{tabular}{|c|c|c|c|c|c|c|}
\hline & \multicolumn{3}{|c|}{ Fut golines } & \multicolumn{2}{|c|}{ Federación } & \multirow[b]{2}{*}{$?^{2}$} \\
\hline & Ítem & $\mathrm{M}$ & DT & $\mathrm{M}$ & DT & \\
\hline \multirow{3}{*}{ Niños } & 1 & 1,28 & 66 & 1,79 & 1,02 & 19,$95 ; p<.001^{*}$ \\
\hline & 2 & 1,39 &, 58 & 1,74 &, 97 & 13,$53 ; p=.004^{*}$ \\
\hline & 4 & 1,50 &, 85 & 1,66 & ,99 & 8,$725 ; p=.406$ \\
\hline \multirow{3}{*}{ Padres } & 1 & 1,20 & 54 & 1,10 &, 33 & 2,$650 ; p=.449$ \\
\hline & 2 & 1,96 &, 73 & 1,94 &, 75 & 8,$325 ; p=.039^{*}$ \\
\hline & 6 & 1,24 & .53 & 1,12 & 45 & 6,$550 ; p=.088$ \\
\hline \multirow{4}{*}{ Formadores } & 1 & 1,71 & 68 & 1,90 & 70 & $805 ; p=.669$ \\
\hline & 2 & 2,06 &, 89 & 2,52 & ,68 & 11,$71 ; p=.008^{*}$ \\
\hline & 6 & 1,65 & 1,17 & 1,71 & 1,19 & 0,$764 ; p=.962$ \\
\hline & & 1,55 & & 1,72 & & \\
\hline
\end{tabular}

M=Media; DT=Desv.típica; ${ }^{2}=$ Chi-cuadrado.

*Di ferencias significativas entre ambos grupos según valor de $p$

Tabla 4

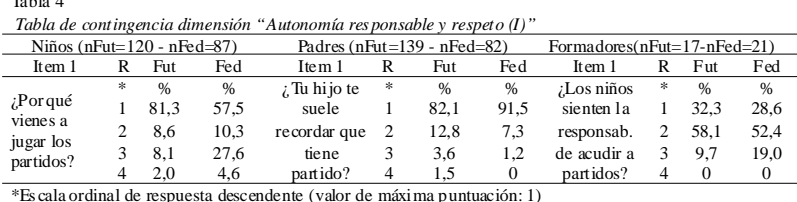

*Es cala ordinal de respuesta descendente (valor de máxi ma puntuación: 1 )

Tabla 5

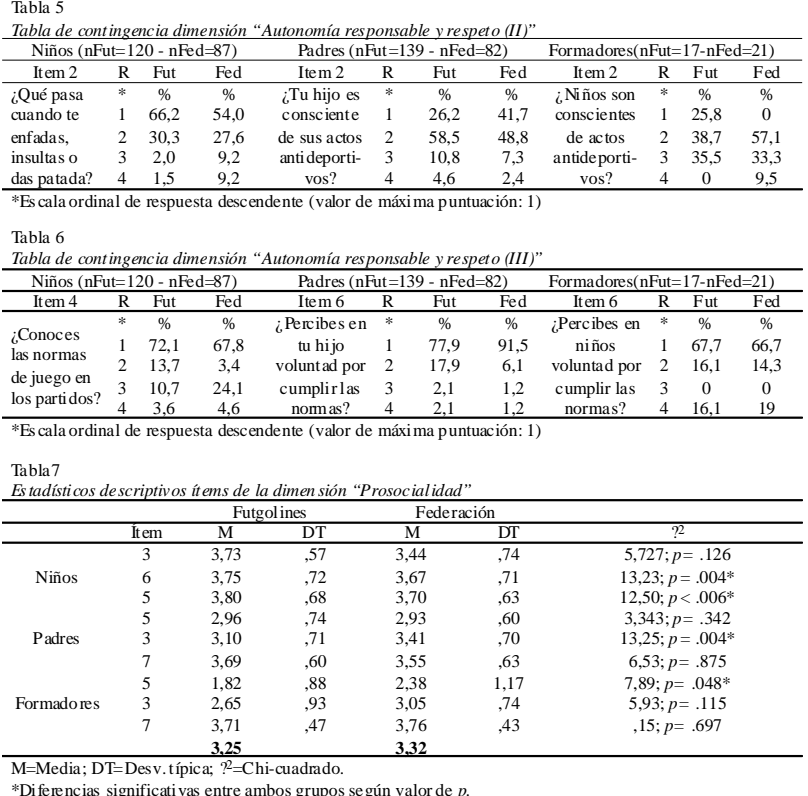

*Di ferencias significativas entre ambos grupos según valor de $p$.

niños en las competiciones (tabla 4). Según el índice $\chi^{2}$ se encuentran diferencias significativas en las respuestas aportadas por el grupo «niños» $(\mathrm{p}<.001)$. Sus opiniones determinan mayor desarrollo de la autonomía responsable en el caso de los participantes en Futgolines, ya que 81.3\% seleccionan la respuesta más positiva, frente a un $57.5 \%$ de los que juegan en la competición federativa. En las respuestas de «padres» y de «formadores» los resultados se muestran bastante equilibrados entre ambos grupos de análisis.

La segunda pregunta trataba de buscar información sobre la percepción de las consecuencias de actos antideportivos. Atendiendo al valor de $p$ en el índice $\chi^{2}$ los resultados muestran diferencias significativas en los tres grupos consultados. En los niños se observa contraste en la conciencia del perjuicio que estos actos suponen para el equipo, más de 12 puntos superior en «Futgolines», mientras que un $18.4 \%$ de los niños de Federación piensan que «no pasa nada» o que «se puede hacer si es para ganar el partido». Por el contrario, entre los formadores destaca la diferencia de más de 20 puntos en cuanto a que un número elevado de ellos en el caso de Federación, opinan que los niños son muy conscientes de las consecuencias de sus actos, mientras que en Futgolines, esa diferencia porcentual se otorga a la respuesta que indica que son «poco» conscientes (tabla 5).

En el tercero de los ítems de esta dimensión, no surgen diferencias estadísticamente significativas en las respuestas de ambos modelos en ninguno de los grupos de consulta, en cuanto al conocimiento por los niños de las normas de juego en los partidos. Se observa claramente una voluntad general por cumplir las normas (tabla 6), ya que más de un $65 \%$ de los consultados eligieron la respuesta más positiva.

\section{Resultados en la dimensión «Prosocialidad»}

La forman los ítems 3,5 y 6 en el cuestionario de niños, y los ítems 3,5 y 7 en padres y formadores. Cuanto más se aproximaran a 4 las respuestas, más se mostraría el desarrollo de los valores analizados. La media de resultados de los respuestas de 276 consultados del modelo «Futgolines», es de 3.25, y de los 191 participantes en el modelo federativo, la media fue de 3.32 (tabla 7). Comparando los resultados obtenidos entre los participantes de los dos modelos de competición analizados, a través del índice $\chi^{2}$, obtenemos:

En el primer ítem se plantea si todos los compañeros son considerados iguales o existen diferencias de trato entre ellos según el nivel de habilidad (pregunta 3 en «niños» y 5 en «padres» $\mathrm{y}$ «formadores»). No se encuentran diferencias significativas entre las variables en las respuestas de «niños» $(\mathrm{p}=.126)$ y «padres» $(\mathrm{p}=.342)$, donde es mayoritaria la opinión de que «todos los compañeros son iguales» (tabla 8). Los datos de los formadores resaltarían diferencias ( $\mathrm{p}=.048$ ), pero muy próximos al umbral de separación independencia/asociación. En este caso, las respuestas están más repartidas, poniendo en tela de juicio esa teórica fiel igualdad entre compañeros.

El segundo de los ítems de esta dimensión, busca predecir la colaboración entre los compañeros dentro de los partidos. Los resultados obtenidos en los grupos «niños» y «padres», muestran diferencias significativas ( $p<.006$ en FUT y $p=.004$ en FED) y exponen que generalmente se pretende un sentido colaborativo del juego (tabla 9). En el grupo «formadores» las respuestas ofrecidas por los participantes en ambos modelos de competición analizados, manifiestan mayor puntuación en los consultados del grupo «Federación» (9.7\% en FUT y 28.6\% en FED seleccionaron la respuesta más positiva).

El tercer ítem valora si existe sentimiento de equipo cuando se marca un gol. Los resultados de «padres» y de «formadores», indican que normalmente los goles se celebran el grupo (tabla 10). Se muestran diferencias estadísticamente significativas $(\mathrm{p}=.006)$ en la respuesta de «niños», siendo mayor el porcentaje de niños del grupo «Futgolines» que consideran que celebran los goles todos juntos (88.5\% FUT; 77\% FED).

\section{Resultados en la dimensión «Satisfacción-diversión»}

Esta categoría fue analizada con las preguntas 7 y 8 en «Niños», 4 y 8 en «Padres» y «Formadores». Las respuestas que predecían mayor nivel de satisfacción-diversión son las que se aproximaban a 4. Entre los 276 participantes en el modelo «Futgolines», se obtienen valores medios de los tres grupos consultados, de 3.72; mientras que de los 191 consultados del formato federativo ofrecieron un valor medio de 3.66 (tabla 11). Como en los casos anteriores, comparamos estos resultados con los obtenidos en las respuestas de los participantes en el formato federativo, mediante la utilización del índice $\chi^{2}$.

En los primeros ítems de esta dimensión se pregunta a los niños si se aburren jugando los partidos y a padres y formadores, si sus hijos les dicen que se han divertido después de los partidos. Los resultados no muestran diferencias significativas en ninguno de los tres grupos de consulta de ambos modelos de competición analizados (tabla 12). En más de un 90\% de los casos se otorgan puntuaciones de valor 3 y 4.

Los segundos ítems se refiere a si los niños acuden contentos a jugar y si quisieran jugar más partidos. No se observan diferencias significativas entre las respuestas de los tres grupos dentro de los dos modelos de competición analizados, siendo siempre despreciables los porcentajes de participantes que dan una respuesta negativa (tabla 13). 
Tabla 8

\begin{tabular}{|c|c|c|c|c|c|c|c|c|c|c|c|}
\hline \multicolumn{4}{|c|}{ Niños (nFut =120 - nFed=87) } & \multicolumn{4}{|c|}{ Padres (nFut=139 - nFed=82) } & \multicolumn{4}{|c|}{ Formadores(nFut=17-nFed=21) } \\
\hline Item 3 & $\mathrm{R}$ & Fut & Fed & Item 5 & $\mathrm{R}$ & Fut & Fed & Item 5 & $\mathrm{R}$ & Fut & Fed \\
\hline \multirow{3}{*}{$\begin{array}{l}\text { ¿Crees que } \\
\text { todos los } \\
\text { compañeros }\end{array}$} & * & $\%$ & $\%$ & & * & $\%$ & $\%$ & \multirow{5}{*}{$\begin{array}{l}\text { ¿Existen } \\
\text { diferencias } \\
\text { de trato por } \\
\text { el nivel de } \\
\text { habilidad? }\end{array}$} & * & $\%$ & $\%$ \\
\hline & 1 & 81,3 & 57,5 & $\begin{array}{l}\text { octamm ent } \\
\text { tu hijo le }\end{array}$ & 1 & 7,1 & 4,9 & & 1 & 32,3 & 33,3 \\
\hline & 2 & 8,6 & 10,3 & e andicion & 2 & 6,1 & 7,4 & & 2 & 38,7 & 9,5 \\
\hline \multirow{2}{*}{$\begin{array}{l}\text { sois igual de } \\
\text { importantes }\end{array}$} & 3 & 8,1 & 27,6 & $\begin{array}{l}\text { el nivel de } \\
\text { enana }\end{array}$ & 3 & 15,2 & 9,9 & & 3 & 3,2 & 14,3 \\
\hline & 4 & 2,0 & 4,6 & habilidad? & 4 & 71,6 & 77,8 & & 4 & 25,8 & 42,9 \\
\hline
\end{tabular}

*Escala ordinal de respuesta des cendente (val or de máxima puntuación: 4 )

$$
\text { Tabla } 9
$$

Tabla de contingencia dimensión "Prosocialidad (II)"

\begin{tabular}{|c|c|c|c|c|c|c|c|c|c|c|c|}
\hline \multicolumn{4}{|c|}{ Niños (nFut $=120-\mathrm{nFed}=87$ ) } & \multicolumn{4}{|c|}{ Padres (nFut=139 - nFed=82) } & \multicolumn{4}{|c|}{ Formadores(nFut=17-nFed=21) } \\
\hline Item 6 & $\mathrm{R}$ & Fut & Fed & Item 3 & $\mathrm{R}$ & Fut & Fed & Item 3 & $\mathrm{R}$ & Fut & Fed \\
\hline \multirow{5}{*}{$\begin{array}{l}\text { En los } \\
\text { partidos, } \\
\text { ¿quién debe } \\
\text { tocar el } \\
\text { balón? }\end{array}$} & * & $\%$ & $\%$ & \multirow{5}{*}{$\begin{array}{l}\text { ¿Percibes un } \\
\text { sentido } \\
\text { colaborativo } \\
\text { en el juego? }\end{array}$} & ${ }^{*}$ & $\%$ & $\%$ & $¿$ ¿Crees que & ${ }^{*}$ & $\%$ & $\%$ \\
\hline & 1 & 5,5 & 3,4 & & 1 & 4,6 & 2,4 & \multirow{4}{*}{$\begin{array}{c}\text { se fomenta } \\
\text { la } \\
\text { consecución } \\
\text { de logros } \\
\text { colectivos? }\end{array}$} & 1 & 16,1 & 0 \\
\hline & 2 & 1,5 & 3,4 & & 2 & 10,8 & 7,3 & & 2 & 9,7 & 23,8 \\
\hline & 3 & 7,5 & 16,1 & & 3 & 58,5 & 48,8 & & 3 & 64,5 & 47,6 \\
\hline & 4 & 85,5 & 77,0 & & 4 & 26,2 & 41,7 & & 4 & 9,7 & 28,6 \\
\hline
\end{tabular}

*Escela ordinal de respuesta des cendente (valorde máxima puntuación: 4)

Tabla de contingencia dimensión "Prosocialidad (III)"

\begin{tabular}{ccc} 
& \\
\hline Niños (nFut $=120-\mathrm{nFed}=87)$ & Padres (nFut $=139-\mathrm{nFed}=82)$ & Formadores(nFut $=17-\mathrm{nFed}=21)$
\end{tabular}

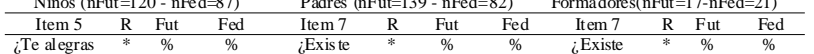

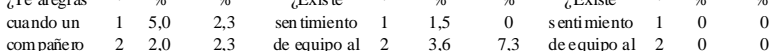

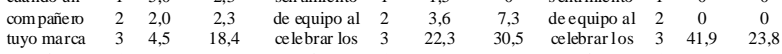

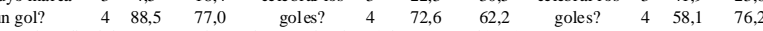

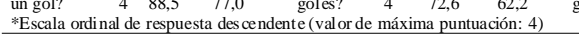

Tabla 11

Estadísticos descriptivos ítems de la dimensión "Satisfacción-d iversión"

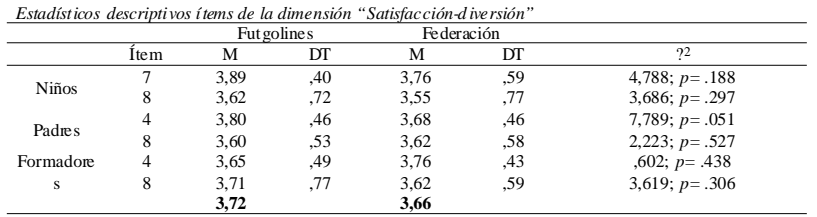

M=Media; DT=Desv. típica; ?2=Chi-cuadrado.

*Diferencias significativas entre amb os grupos según valor de $p$

Tabla 12
Tabla de contingencia dimensión "Satisfacción-diversión (I)"

\begin{tabular}{|c|c|c|c|c|c|c|c|c|c|c|c|}
\hline \multicolumn{4}{|c|}{ Niños (nFut $=120$ - nFed=87) } & \multicolumn{4}{|c|}{ Padres (nFut=139 - nFed=82) } & \multicolumn{4}{|c|}{ Formadores(nFut $=17-\mathrm{nFed}=21$ ) } \\
\hline Item 7 & $\mathrm{R}$ & Fut & Fed & Item 4 & $\mathrm{~B}$ & Fut & Fed & Item 4 & & Fut & Fed \\
\hline & $*$ & $\%$ & $\%$ & Después de & * & $\%$ & $\%$ & ¿Los niños & * & $\%$ & $\%$ \\
\hline $\mathrm{¿Te}$ aburres & 1 & 1,0 & 2,3 & & 1 & 0,5 & 0 & & 1 & 0 & 0 \\
\hline jugando los & 2 & 0,5 & 1,1 & partidos, ¿tu & 2 & 0,5 & 0 & se divierten & 2 & 0 & 0 \\
\hline partidos? & 3 & 7,5 & 14,9 & $\begin{array}{l}\text { hijo se ha } \\
\text { divertido? }\end{array}$ & 3 & 15,2 & 31,7 & $\begin{array}{l}\text { jugando los } \\
\text { partidos }\end{array}$ & 3 & 22,6 & 23,8 \\
\hline & 4 & 91,0 & 81,6 & & & 83,2 & 68,3 & & 4 & 77,4 & 76,2 \\
\hline
\end{tabular}

Tabla 13

Tabla de contingencia dimensión "Satisfacción-diversión (II)"

\begin{tabular}{|c|c|c|c|c|c|c|c|c|c|c|c|}
\hline \multicolumn{4}{|c|}{ 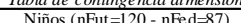 } & \multicolumn{4}{|c|}{ 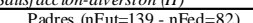 } & \multicolumn{4}{|c|}{ ormadores(nFut=17-nFed=21) } \\
\hline Item 8 & $\mathrm{R}$ & Fut & Fed & Item 8 & $\mathrm{R}$ & Fut & Fed & Item 8 & $\mathrm{R}$ & Fut & Fed \\
\hline ¿Te gustaría & * & $\%$ & $\%$ & ${ }_{i \text { Notas a tu }}$ & * & $\%$ & $\%$ & & * & $\%$ & $\%$ \\
\hline jugar & 1 & 1,0 & 2,3 & hijo más & 1 & 0 & 1,2 & Los niños & 1 & 0 & 0 \\
\hline tidos & 2 & 0,5 & 1,1 & contents & 2 & 1,5 & 1,2 & acuden & 2 & 3,2 & 4,8 \\
\hline todos los & 3 & 7,5 & $\begin{array}{l}1,1 \\
14,9\end{array}$ & cuando tiene & 3 & $\begin{array}{l}1,0 \\
35,0\end{array}$ & $\begin{array}{l}1,2 \\
31,7\end{array}$ & contentos a & 3 & $\begin{array}{l}3,2 \\
9,7\end{array}$ & $\begin{array}{r}28,6 \\
28,6\end{array}$ \\
\hline días? & 4 & 91,0 & 81,6 & partido? & 4 & 63,5 & 65,9 & los partidos? & & 87,1 & 66,7 \\
\hline
\end{tabular}

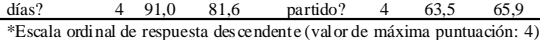

\section{Discusión}

La competición deportiva en esencia contribuye a esforzarnos, a progresar, y cuando está bien orientada, nos proporcionará desarrollos a nivel de personalidad (López, 2005; Durán, 2013), y como se deduce de los resultados obtenidos, en general nos lleva a prosperar en la asimilación y aplicación de los valores socio-educativos analizados.

Haciendo una valoración más detenida analizando cada una de las dimensiones, comprobamos que los encuestados consideran que la competición deportiva es una fuente de desarrollo de la autonomía responsable y el respeto. En los resultados se muestra que existe conocimiento y respeto del reglamento por parte de los niños y son conscientes de las repercusiones que su incumplimiento acarrea para ellos y para su equipo. En la literatura encontramos opiniones a favor y en contra de este planteamiento. Barberá (2001) considera que los niños pequeños no son responsables porque no pueden calcular las consecuencias de sus acciones. Piaget (1932) coincide con esta opinión, alertando de la presencia de un nivel de egocentrismo y Kohlberg (1982), señala que en este periodo los niños regulan su conducta con la única intención de evitar castigos y lograr recompensas (definitoria de la fase de heteronomía moral). En oros estudios posteriores (Guijo, 2002; Monsalvo \& Guaraná, 2008) se comprueba que niños de la segunda etapa de Educación Infantil tienen comportamientos que manifiestan cierto nivel de adopción desde el punto de vista del otro, considerándose como indicador fundamental del desarrollo prosocial y de la responsabilidad.
Fueron halladas diferencias estadísticas significativas entre los participantes de «Futgolines» y de «Federación», en la dimensión «Autonomía responsable y respeto». En primer lugar llama la atención que uno de cada cinco niños de «Federación» declaran que juegan por imposición o condicionamiento de sus padres. También sucede cuando se les pregunta a los niños sobre las consecuencias ante determinados actos antideportivos, donde casi un $20 \%$ de los encuestados de «Federación» dicen que «no pasa nada» o que «se puede hacer si es para ganar el partido», justificando así la realización de actos antideportivos, por un 3,3\% de los que lo piensan en Futgolines. Entre los formadores se corrobora esta diferencia ya que, lo significativo se encuentra en que ninguno de los consultados del modelo federativo piensa que los niños son «muy conscientes» de las consecuencias de sus malos actos en los partidos, frente a más de un $40 \%$ de los consultados de «Futgolines». Esto se debe seguramente por un lado a que desde el formato «Futgolines» se promueve la capacidad de diálogo explicativo de los formadores y por otro a que al conocer la normativa, saben que si cometen algún acto que el controlador del partido considera que no es adecuado, se resta un punto al equipo. Esta idea que señala al sistema de competición como claro condicionante de los comportamientos de los participantes es compartida por varios autores (González-Suárez \& Otero, 2005; Latorre et al., 2009; Tuero, Zapico \& González, 2012; Sánchez, Yagüe \& Molinero, 2012), que explican que la clave del problema reside en la consideración de la práctica deportiva desde la propuesta que ofrecen las federaciones regionales comoúnica referencia válida, en las que todo gira alrededor de la búsqueda del modelo de campeón y la práctica puramente competitiva para conseguirlo.

La autorregulación personal y el respeto al otro analizada en la primera parte, precisa de cierto desarrollo de la capacidad empática. Diversas investigaciones han hallado relaciones altamente significativas entre empatía y conducta prosocial (McMahon, Wernsman \& Parnes, 2006; De la Fuente, Peralta \& Sánchez, 2009; Gutiérrez, Escartí \& Pascual, 2011). En los datos de nuestro estudio se muestra una clara tendencia hacia la prosocialidad en ambos modelos de competición. Estos resultados coinciden con opiniones que explican que en un buen engranaje de funcionamiento colectivo surgirán relaciones a las que se suma lo emocional, originándose la amistad (Gutiérrez et al., 2011; Valdemoros, Ponce de León, Ramos \& Sanz, 2011; Del Prette et al., 2013). Se obtienen porcentajes más abultados entre los consultados de «Federación» sobre la realización de un juego más colectivo. Además, llama la atención que, aunque «niños» y «padres» consideran que todos los compañeros del equipo son iguales, los «formadores» reconocen diferencias de trato evidentes a favor de los niños que juegan mejor, siendo a su vez esta circunstancia mayor entre los encuestados de «Futgolines».

La «adecuada orientación» de la competición deportiva que se persigue a lo largo de toda la investigación, se concreta en la implicación hacia la tarea y la motivación intrínseca de los participantes. Esto se pone de relieve en los resultados relativos por un lado al compromiso voluntario de práctica sin coacción exterior y por otro, el alto grado de satisfacción y diversión mostrado por los niños y niñas consultados y corroborado por sus padres y formadores. Además, no se observan diferencias ostensibles entre las opiniones de aquellos participantes en uno o en otro formato competitivo. Aunque se percibe que la mayoría de los que seleccionan la opción 4 en las preguntas realizadas normalmente es mayor en «Futgolines», algunos encuestados advierten que, tanto en uno como en otro formato, existe un pequeño número de niños (siempre por debajo del 6\%) que no se divierten jugando los partidos. Luego, se podría determinar que aunque el nivel de alta satisfacción y diversión es contundentemente mayoritario, no es unánime. Por otra parte, aunque la satisfacción/diversión se demuestra de manera más intensa en Futgolines, las diferencias entre ambos formatos son pequeñas y no compartidas por todos los grupos de sujetos consultados.

La relación encontrada entre los niveles de satisfacción-diversión por la práctica, las conductas prosociales, la autonomía responsable y el respeto está en consonancia por lo planteado desde la Teoría de la Autodeterminación(Ryan \& Deci, 2000). Sánchez-Oliva, Leo, Sánchez, 
Gómez y García-Calvo (2011) establecen que a mayores niveles de autodeterminación, más comportamientos prosociales aparecen. Concluyeron que los jugadores que muestran una motivación más intrínseca (compromiso de un deportista con una actividad por placer, disfrute y satisfacción que le produce) tienden a mostrar mayores intenciones de realizar comportamientos prosociales y mayor probabilidad de realización de dichos comportamientos.

En los datos obtenidos podría percibirse la diferente intencionalidad formativa que erigen ambos formatos analizados. «Futgolines» es planteado como una propuesta educativa, frente a la formación fundamentalmente deportiva hacia la que se orienta el formato de competición federativo (Manrique, López, Monjas, Barba \& Gea, 2011; Reverter, Plaza, Jové \& Mayolas, 2012; Lledó, Martínez \& Huertas, 2014).

Una de las limitaciones del estudio reside en que la muestra consultada se limita a una zona concreta en la que se aplican los dos modelos de competición analizados. Además, el estudio podría completarse con la utilización de pruebas cualitativas como entrevistas o grupos de discusión. Por otra parte, debe tenerse en cuenta en los resultados la media de edad más elevada en los niños consultados de «Federación» (M=6,85), frente a los participantes de «Futgolines» $(\mathrm{M}=5,99)$, por la posible influencia del nivel madurativo.

En el presente estudio se muestran datos que hacen pensar que la participación en competiciones deportivas adecuadamente orientadas puede tener consecuencias a nivel global. Estas relaciones generadas entre los miembros de un equipo, muestra de una adecuada convivencia (Vizcarra, Macazaga \& Rekalde, 2006; Gutiérrez et al., 2011), podría tener trascendencia a otros contextos de la vida cotidiana, avanzando en civismo (Zurbano, 2001; Valdemoros et al., 2011), produciéndose así una mejor vida en sociedad. Sin embargo, vemos necesario un estudio longitudinal más profundo para llegar a esta conclusión, por lo que planteamos aquí otra posible línea de investigación futura.

\section{Conclusiones}

Según la opinión de los consultados, la participación en competiciones deportivas de los formatos analizados estimula el desarrollo de los valores educativo-sociales analizados: autonomía responsable y respeto, prosocialidad y satisfacción-diversión.

Los resultados no son siempre coincidentes entre los participantes de uno y otro modelo y denotan la posible diferencia en la intencionalidad formativa de uno y otro formato, más próximo a lo educativo en «Futgolines» y a lo meramente deportivo en el modelo federativo.

- En la dimensión «autonomía responsable y respeto» observamos en los tres grupos de consulta, que existe relación entre el modelo de competición en el que se participa y la justificación de actos antideportivos con pretensiones resultadistas. Los consultados del modelo federativo encuentran mayor justificación de los actos antideportivos si es para ganar el partido, y se percibe mayor coacción sometida por sus padres incitándoles a la participación en dichas competiciones.

- Dentro de la categoría «Prosocialidad», se considera que en el modelo federativo se realiza un juego más colectivo. Además, los formadores advierten que a nivel social los niños tienen predilección por los compañeros que juegan mejor, siendo más acentuado entre los niños de «Futgolines».

- Existe un elevado nivel de satisfacción-diversión percibido con la participación en las competiciones, independientemente del formato bajo el que se compita, aunque los resultados más positivos son ligeramente superiores en los niños de «Futgolines».

\section{Referencias}

Barberá, V. (2001). Cómo educar en la responsabilidad. Madrid: Santillana. Berengüí Gil, R. \& Garcés, E. J. (2007) Valores en el deporte escolar: estudio con profesores de Educación Física. Cuadernos de Psicología del Deporte, 7(2), 90-103.

Buendía, L., Colás, P. \& Hernández, F. (2003). Métodos de Investigación en Psicopedagogía. Madrid. Mc Graw-Hill.

Carreras, L.; Eijo, P.; Estany, A.; Gómez, M. T.; Guich, R.; Mir, V. et al. (1995). Cómo educar en valores. Materiales, textos, recrusos y técnicas. Madrid: Narcea.
Chappuis, R., \& Thomas, R. (1989) El equipo deportivo. Barcelona: Paidós-MEC.

Celina, H. \& Campo, A. (2005) Aproximación al uso del coeficiente alfa de Cronbach. Revista colombiana de psiquiatría, XXXIV (4), 572 580 .

Consejo Superior de Deportes (2010) Plan integral para la actividad física y el deporte.

De la Fuente, J., Peralta, F.J., \& Sánchez, M.D. (2009). Autorregulación personal y percepción de los comportamientos escolares desadaptativos. Psicothema, 15, 548-554.

Del Prette, Z. A. P.; Domeniconi, C.; Amaro, L.; Benítez, P.; Laurenti, A., \& Del Prette, A. (2013). La tolerancia y el respeto a las diferencias: efectos de una actividad educativa en la escuela. Apuntes de Psicología, 31 (1), 59-66.

Durán, J. (2013). Ética de la competición deportiva: valores y contravalores del deporte competitivo. Materiales para la historia, 11, 89-115.

Erikson, E. (1971) Infancia e sociedad. Rio de Janeiro: Zahar editores. González-Suárez, M., \& Otero, M. (2005). Actitudes de los padres ante la promoción de la actividad física y deportiva de las chicas en edad escolar. Cuadernos de Psicología del Deporte, 5, 173-195.

Guijo, V. (2002). Estudio Multifactorial de la Conducta Prosocial en niños de cinco y seis años (Tesis doctoral). Universidad de Burgos, Burgos.

Gutiérrez, M., Carratalá, V., Guzmán, J. F., \& Pablos, C. (2010) Objetivos y manifestaciones de Valores sociales y personales en el deporte juvenil según deportistas, padres, entrenadores y gestores. Apunts. Educación física y deportes, 101, 57-65.

Gutiérrez, M.; Escartí, A. \& Pascual, C. (2011) Relaciones entre empatía, conducta prosocial, agresividad, autoeficacia y responsabilidad personal y social de los escolares. Phycothema, 23(1), 13-19.

Gutiérrez M. \& Pilsa, C. (2006) Orientaciones hacia la deportividad de los alumnos de educación física. Apunts. Educación Física y Deportes. 86, 86-92.

Hidalgo Montero, M. (2008, junio). Abandono deportivo escolar: más temprano que tarde. Lecturas: Educación Física y Deporte. Revista Digital, 121. http://www.efdeportes.com/efd121/abandono-deportivo-escolar.htm [consulta 12 de agosto de 2012].

Kohlberg (1982). Moral stages and moralizations. En T. Lickona (Ed). Moral development and behavior. Trad. Cast. Infancia y Aprendizaje, 20, 33-51.

Jiménez, B., López, V. M. \& Manrique, J.C. (2014). Evaluación comparativa de resultados de un programa municipal de deporte escolar. Retos. Nuevas tendencias en Educación Física, Deporte y Recreación, 26, 15-20.

Juan-Llamas, C. (2015) Diseño y validación de un cuestionario sobre la forma de trabajo de los instructores de clases colectivas. Retos, 27, 19-23.

Latorre, P.A.; Gasco, F.; García. M.; Martínez, R. M.; Quevedo, O.; Carmona, F.J.; Rascón P.J.; Romero, A.; López, G.A., \& Malo, J. (2009). Analysis of the influence of the parents in the sports promotion of the children. Journal of Sport and Health Research, 1(1): $12-25$.

Lawshe, C. H. (1975). A quantitative approach to content validity. Personnel Psychology, 28, 563-575.

Lledó, E.; Martínez, G. y Huertas, F. (2014). Perfil del entrenador de fútbol en la etapa escolar en escuelas de clubes de élite de la Comunitat Valenciana. Cultura, Ciencia y Deporte 25 (9), 57-68.

López, M.L. (2005) Valor educativo del juego competitivo en un entorno marginal. Los «moinantes» aprenden a competir y cooperar. Tándem Didáctica de la Educación Física, 19. 73-78.

López, E.; Pérez, A., \& Ramos, G. (2011). Modelos complementarios al Análisis Factorial en la construcción de escalas ordinales: un ejemplo aplicado a la medida del Clima Social Aula. Revista de Educación, 354, 369-397.

Mandado, A. \& Díaz, P. (2004) Deporte y educación: Pautas para hacer compatible el rendimiento y el esarrollo integral de los jóvenes deportistas. Revista de Educación, 335, 35-44.

Manrique, J. C.; López, V.M. Monjas, R.; Barba, J.J., y Gea, J.M. (2011) Implantación de un proyecto de transformación social en Segovia (España): desarrollo de un programa de deporte escolar en toda la ciudad. Apunts. Educación Física y Deportes, 105, 58-66.

Marina, J. A. (2004). Aprender a vivir. Barcelona: Ariel.

McMahon, S., Wernsman, J., \& Parnes, A. (2006) Understanding prosocial behaviour: The impact of empathy and gender among African American adolescents. Journal of Adolescent Health, 39, 135-137. 
Monsalvo, E., \& Guaraná, R. (2008). El valor de la responsabilidad en los niños de educación infantil y su implicación en el desarrollo de comportamientos prosociales. Revista Ibero-Americana de Educación, 47 (2).

Morales, M. T. \& Arias-Estero, J.L. (2015). Diferencias entre el juego 7 vs.7 y el 4 vs. 4 en el balonmano escolar en relación al rendimiento, percepción del esfuerzo y la intencionalidad de la práctica. Retos, 27, 34-39.

Piaget, J. (1932) El criterio moral en el niño. Barcelona: Fontanella.

Piñar, M. I.; Cárdenas, D.; Miranda, T., \& Torre, E. (2008). Factores que afectan al aprendizaje durante la competición e influyen en la formación del jugador de minibasket. Habilidad Motriz, 31, 5-15.

Reverter, J.; Plaza, D.; Jové, M.C., y Mayolas, M.C. (2012) Influencia de los técnicos en el deporte extraescolar. El caso de la ciudad de Torrevieja. Retos. Nuevas tendencias en Educación Física, Deporte y Recreación, 22, 76-80.

Ruiz, J.V. (2004) Pedagogía de los valores en la Educación Física. Madrid: Editorial CCS.

Ryan, R.M. \& Deci, E.L. (2000). Self-determination theory and the facilitation of intrínsico motivation, social develpment and wellbeing. American Psychologist, 55(1), 68-78.

Sánchez, J.; Yagüe, J. M., \& Molinero, O. (2012). Estudio del nivel de diversión generado por la aplicación de un programa de entrenamiento técnico y otro táctico en futbolistas jóvenes. Cuadernos de Psicología del Deporte 13 (1), 95-102.

Sánchez-Oliva, D.; Leo, F. M.; Sánchez, P.A.; Gómez, F.R., \& GarcíaCalvo, T. (2011). Teoría de autodeterminación y comportamientos prosociales en jóvenes jugadores de fútbol. Apunts. Educación Física y Deportes, 103, 32-38.

Sánchez-Oliva, D.; Leo, F. M.; Sánchez-Miguel, P. A.; Amado, D., \& García-Calvo, T. (2012). Antecedentes motivacionales de los comportamientos prosociales y antisociales en el contexto deportivo. Revista Internacional de Medicina y Ciencias de la Actividad Física y el Deporte, 12 (46), 253-270.

Scheaffer, R.; Mendenhall, W., \& Lyman Ott, R. (2006). Elementos del muestreo. Madrid. Paraninfo.

Schwartz, S. H.; Verkasalo, M.; Antonovsky, A., \& Sagiv, L. (1997) Value priorities and social desirability: much substance, some style. British
Journal of Social Psychology, 36, 3-18.

Tuero, C.; Zapico, B., \& González, R. (2012) Deporte en edad escolar y agentes sociales: estudio preliminar sobre la relación entre familia, monitores y deportistas en la provincia de León. Retos. Nuevas tendencias en Educación Física, Deporte y Recreación, 21 (34-37).

Valdemoros, M. A.; Ponce de León, A.; Ramos, R. \& Sanz, E. (2011) Pedagogía de la convivencia y la educación no formal: un estudio desde el ocio físico-deportivo, los valores y la familia. European Journal of Education and Psichology, 4(1), 33-49.

Valero, A.; Conde, A.; Delgado, M., \& Conde, J.L. (2004). Construcción y validación de un cuestionario de diversión y adherencia hacia la práctica del atletismo en la educación primaria. Revista Española de Educación Física y Deportes, 1, 119-130.

Veroz, R.; Parra, R.; Masegosa, J.; García, P., \& Martínez, L. (2010). Modelo de competición de fútbol para categoría pre-benjamín adaptado a las capacidades de los participantes, favoreciendo el desarrollo de adecuados valores deportivos y educativos. Revista Abfútbol, 46, 71-82.

Vizcarra, M.J.; Macazaga, A. M., \& Rekalde, I. (2006) «¿Con qué deporte escolar sueñan las familias?». Apunts. Educación Física y deportes, 86, 97-107.

Zurbano, J.L. (2001). Educación para la convivencia y para la paz. Educación Primaria. Pamplona: Gobierno de Navarra. Departamento de Educación y Cultura.

\section{(Footnotes)}

Se modifican las condiciones básicas de juego (partidos de 4 contra 4, en campos y porterías más pequeñas) y el sistema de puntuación propio del modelo federativo imperante para que la victoria no sea sólo consecuencia directa de méritos deportivos. Algunas aportaciones concretas son la consecución de puntos sólo si se abrazan todos los compañeros del equipo tras conseguir el gol, se sumarán dos puntos si los 4 jugadores del equipo participan en la jugada, se primará la consecución de al menos un gol por parte de todos los compañeros o se penalizarán actos antideportivos o faltas de respeto, disminuyen puntos en el marcador (Veroz, Parra, Masegosa, García \& Martínez, 2010).

${ }^{2}$ En algunos casos, debido a su corta edad, el procedimiento de administración se realizó con la lectura de los ítems por parte del investigador principal del trabajo y la anotación de las respuestas.

${ }^{3}$ Dichos datos forman parte de un estudio más amplio que el presentado en este documento, por lo que no serán tenidos en cuenta en este momento.

\begin{tabular}{|c|c|c|}
\hline \multirow{2}{*}{$\begin{array}{l}\text { Anexo } \\
\text { Cuestionario "Deporteduca" }\end{array}$} & & \\
\hline & \multicolumn{2}{|c|}{ INF ORMACIÓN INICIAL: Edad del niño/a: ----____ Modelo de competición:______ Años jugando con este formato: __ } \\
\hline Destinatario: NIÑOS & PADRES & FORMADORES \\
\hline $\begin{array}{l}\text { 1) ¿Por qué vienes a jugar los partidos? } \\
\text {-Porque quiero yo, si empre me acuerdo del partido. } \\
\text {-Vengo porque quiero yo, pero a veces no me acuerdo. } \\
\text {-A mis padres les gusta verme ju gar. } \\
\text {-Porque mis padres me obligan. }\end{array}$ & $\begin{array}{l}\text { 1) ¿Tu hijo/a te suele recordar que tien e partid o? } \\
\text {-Siempre. } \\
\text {-Frecuentemente. } \\
\text {-Casi nunca. } \\
\text { - Nunca. }\end{array}$ & $\begin{array}{l}\text { 1) ¿Crees que los niños sienten la responsabilidad de tener que } \\
\text { acudir a los partidos? } \\
\text { aMucho. } \\
\text { ZBastante. } \\
\text { DPoco. } \\
\text { aNada. }\end{array}$ \\
\hline $\begin{array}{l}\text { 2) Cuando en un partido te en fadas, insultas o das una patada, } \\
\text { ¿qué crees que pasa? } \\
\text {-Es malo para mi equipo. } \\
\text {-Sólo es malo para mí, a mi equipo no le pasa nada. } \\
\text {-No pasa nada. } \\
\text {-Se puede hacer si es para ganar el partido. }\end{array}$ & $\begin{array}{l}\text { 2) } ¿ \text { Consideras que tu hijo/a es consciente de las consecuencias de } \\
\text { los actos antid quortivos que pueda cometer en un partido? } \\
\text { - Much o. } \\
\text { - Bastante. } \\
\text { - Poco. } \\
\text { - Nada. }\end{array}$ & $\begin{array}{l}\text { 2) } ¿ \text { Consider as que los niños son conscientes de las consecuencias } \\
\text { de los actos antideportivos que puedan cometer en un partido? } \\
\text { DMucho. } \\
\text { aBastante. } \\
\text { DPoco. } \\
\text { aNada. }\end{array}$ \\
\hline $\begin{array}{l}\text { 3) ¿Crees que todos los compañer os del equipo sois igual de } \\
\text { importantes? } \\
\text { ¿Son más importantes los que juegan mejor. } \\
\text { ¿Son más importantes los que marcan más goles. } \\
\text { ¿Son más importantes los que se portan mejor. } \\
\text { ¿Sí. }\end{array}$ & $\begin{array}{l}\text { 3) ¿Percibes un sentido colaborativo en el juego? } \\
\text { ¿No, suele cogerel balón un niño/a y apenas pasa el balón. } \\
\text { aNormal mente no. } \\
\text { ZSí, los chicos/as intentan pasar para que todos jueguen. } \\
\text { QSí, claramente se percibe la colaboración entre todos los } \\
\text { compañeros/as. }\end{array}$ & $\begin{array}{l}\text { 3) ¿Crees que se fomenta la consecución de logros colectivos? } \\
\text { वNo, suelen producise individual idades. } \\
\text { UNormal mente no. } \\
\text { DSí, los chicos /as intentan pasar para que todos jueguen. } \\
\text { USí, claramente se desarrolla un juego colectivo. }\end{array}$ \\
\hline $\begin{array}{l}\text { 4) ¿Conoces las normas de juego en los partidos? } \\
\text { ¿Sí, todas. } \\
\text { ¿Creo que sí. } \\
\text { ¿Algunas, pero no todas. } \\
\text { ¿No, las normas dan igual. }\end{array}$ & $\begin{array}{l}\text { 4) Cuando pregu ntas a tu hijo/a después de los partidos, éélella } \\
\text { se ha divertido? } \\
\text { •Nada. } \\
\text {-Poco. } \\
\text { •Bastante. } \\
\text { •Much o. }\end{array}$ & $\begin{array}{l}\text { 4) ¿Ves que los ni ños se divierten jugando los partidos? } \\
\text { •Nada. } \\
\text { •Poco. } \\
\text { •Bastante. } \\
\text { वMucho. }\end{array}$ \\
\hline $\begin{array}{l}\text { 5) ¿Te alegras cuan do un compañero tuyo marca un gol? } \\
\text { - No, sólo me alegro cuando los marco yo. } \\
\text {-Sólo celebra el gol el que lo marca. } \\
\text { •Sí, lo colebro muy contento. }\end{array}$ & $\begin{array}{l}\text { 5) ¿Crees que en el aspecto social, a tu hijo/a le condiciona el nivel } \\
\text { de habilidad de sus companéros/as? } \\
\text { aSí, ve más importantes a los/as que son mejores jug ando al fútbol. } \\
\text { DSí, intenta que los mejores sean sus ami gos } \\
\text { aNo, para él/ella los más importantes son sus amigos/as. } \\
\text { aNo, para él/el la todos sus compañeros/as son igual de importantes. }\end{array}$ & $\begin{array}{l}\text { 5) ¿Crees que entre los niños existen diferencias de trato } \\
\text { evidentes dependiendo de su nivel de habilidad? } \\
\text { •Ś, ven más importantes a los que son mejo res jugando al fútbol. } \\
\text {-Sí, todos/as quieren ser amigos/as de los/as que mejor juegan. } \\
\text { •-No, el niv el de habilidad no influye en esto. } \\
\text { •No, para ellos/as todos los compañ eros/as son igual de importantes. }\end{array}$ \\
\hline $\begin{array}{l}\text { 6) En los partidos, ¿quién debe tocar el balón? } \\
\text {-Sólo los mejores. } \\
\text {-No importa. } \\
\text {-Cualquiera que esté bien colocado. } \\
\text {-Todos/as, para poder jugar todos/as. }\end{array}$ & $\begin{array}{l}\text { 6) ¿Percibes en tu hijo/a vol untad por aumplir las reglas del } \\
\text { juego? } \\
\text {-Ve muy importante cumplir las normas de juego. } \\
\text {-Ni las conoce, sólo le importa jugar. } \\
\text { - Ni las conoce, sólo le importa ganar. } \\
\text { - No les importa hacer trampas si es para ganar. }\end{array}$ & $\begin{array}{l}\text { 6) ¿Percibes en los niños de tu equipo vol untad por aumplir las } \\
\text { norma s? } \\
\text {-Ven muy importante cumplir las normas de juego. } \\
\text {-Ni las conocen, sólo les importa jugar. } \\
\text {-Ni las conocen, sólo les importa ganar. } \\
\text { •No les importa hacer trampas si es para ganar. }\end{array}$ \\
\hline $\begin{array}{l}\text { 7) ¿Te aburres jugando los partidos? } \\
\text { •Sí, siempre. } \\
\text { - Bastantes veces. } \\
\text {-Algunas veces. } \\
\text { • No, nunca. }\end{array}$ & $\begin{array}{l}\text { 7) ¿Crees que existe sentimiento de equipo a la hora de celebrar } \\
\text { los goles? } \\
\text { aNo, parece que la consecución del gol es mérito individual. } \\
\text { Q Algunas veces. } \\
\text { DHabitual mente sí. } \\
\text { aSí, siempre lo celebran en grupo. }\end{array}$ & $\begin{array}{l}\text { 7) ¿Crees que exis te sentimi en to de equipo a la hora de celebrar } \\
\text { los goles? } \\
\text { aNo, parece que la consecución del gol es mérito individual. } \\
\text { DAlgunas veces. } \\
\text { DHabitual mente sí. } \\
\text { aSí, siempre lo cel ebran en grupo. }\end{array}$ \\
\hline $\begin{array}{l}\text { 8) ¿Te gustaría jugar partidos todos los días? } \\
\text {-No. } \\
\text {-Me da igual (se muestra indi ferente). } \\
\text {-Sí, pero también me gustan otras cosas. } \\
\text { •Sí, jugar partidos es lo que más me gusta. }\end{array}$ & $\begin{array}{l}\text { 8) ¿Notas a tu hijo/a más contento/a cuando tiene partido? } \\
\text { - No, le noto más disgustado. } \\
\text { - No, se muestra indiferente. } \\
\text { - Nomalm ente sí. } \\
\text {-Sí, mucho más. }\end{array}$ & \begin{tabular}{|l|} 
8) ¿Crees que los ni ños acuden contentos a jugar los partidos? \\
-No, a algunos les noto disgustados. \\
•No, algunos se muestran indiferentes. \\
-Sí, salvo algunas excepciones. \\
- Sí, todos/as. \\
\end{tabular} \\
\hline
\end{tabular}

